\section{MS13-P11 Probing the structural requisites for enzymatic flavin-N5-oxide formation}

Raspudin Saleem Batcha ${ }^{1}$, Robin Teufel ${ }^{1}$

1. University of Freiburg, Center for Biological Systems Analysis (ZBSA), Habsburgerstr. 49, 79104 Freiburg, Germany and Faculty of Biology, Schänzlestr. 1, 79104 Freiburg, Germany

email: raspudin.saleem.batcha@zbsa.uni-freiburg.de

The flavin-dependent monooxygenases are among the most intensively studied enzymes that accomplish a variety of redox reactions such as the monooxygenation of organic substrates. Our understanding of the enigmatic reaction of enzyme-bound reduced flavin with oxygen is incomplete, as underlined by recent studies on the biosynthesis of the bacterial polyketide antibiotic enterocin. Here, the flavin-dependent monooxygenase EncM acts as the key tailoring enzyme by oxygenating the enterocin precursor with an unusual flavin-N5-oxide intermediate. Before then, it was presumed that all flavin-dependent monooxygenases employ the flavin-C4a-hydroperoxide as the oxygenating species. We aim to identify the structural prerequisites for the generation of this novel flavin redox state and investigate EncM variants as well as homologous flavoproteins (native and variants), which do not naturally form the flavin-N5-oxide. With the help of the crystal structure of EncM and in silico docking methods, a model for the interaction of $\mathrm{O}_{2}$-binding pocket was predicted and various EncM mutants were generated. Moreover, protein crystals suitable for X-ray crystallographic studies were obtained for several EncM mutants allowing the assessment of the structural changes. Currently, we aim to engineer a similar $\mathrm{O}_{2}$-binding pocket in a structurally related flavoenzyme that does not naturally form the flavin-N5-oxide. Ultimately, this may allow to address some fundamental questions of the flavin cofactor's oxygen reactivity and thus facilitate future rational engineering of flavoproteins with the aim of fine-tuning their oxygen reactivity and product formation.

Keywords: crystallography
MS13-P12 Crystal structure of a fast recovering photoreceptor protein PpSB2-LOV: elucidation of kinetic properties and signal transduction mechanism

Vladimir Arinkin ${ }^{1}$, Joachim Granzin ${ }^{1}$, Ulrich Krauss ${ }^{2}$, Karl-Erich Jaeger $^{2}$, Dieter Willbold ${ }^{1}$, Renu Batra-Safferling ${ }^{1}$

1. Institute of Complex Systems, ICS-6: Structural Biochemistry, Forschungszentrum Jülich, D-52428 Jülich, Germany

2. Institute of Molecular Enzymtechnology, Heinrich-Heine-Universität Düsseldorf, D-52426 Jülich, Germany

email: v.arinkin@fz-juelich.de

LOV (light-oxygen-voltage) photoreceptor proteins control multiple light-dependent cellular responses in prokaryotes and eukaryotes. Mostly LOV domains are linked to signaling and regulatory domains like histidine and serine/threonine kinases, helix-turn-helix DNA binding domain, sigma factor regulators and many others. Interestingly, $13 \%$ of all LOV proteins are present as single domains [1]. LOV domains bind flavin chromophores non-covalently like flavin mononucleotide, riboflavin and flavin adenine dinucleotide. Upon blue light absorption, an adduct is formed between $\mathrm{C} 4 \mathrm{a}$ atom of flavin chromophore and the sulfur atom $\mathrm{S} \gamma$ of a conserved cysteine. In this light state, adduct is stable at a timescale ranging from seconds to days before the recovery of initial dark state [2]. LOV proteins also show considerable variation in the structure and length of $\mathrm{N}$ and C-terminal extensions, which mimic the signal transduction linker regions.

PpSB2-LOV from Pseudomonas putida KT2440 has a fast dark recovery time of $\tau=2.3 \mathrm{~min}$ at $20^{\circ} \mathrm{C}$, in contrast to $\tau=2471 \mathrm{~min}$ of the homolog PpSB1-LOV [3]. In this work, the dark state crystal structure of PpSB2-LOV was determined at $1.9 \AA$ resolution that revealed a conserved LOV core domain, and a dimer interface mediated via the $\mathrm{N}$ - and $\mathrm{C}$-terminal helices. In a comparative analysis, we show differences at single amino acid level comparing PpSB2-LOV with PpSB1-LOV protein to propose the molecular mechanism responsible for variation in the dark recovery time. Furthermore, we present evidence for recently proposed "rotary switch" signal transduction mechanism, based on the fully-adapted light and dark structures of PpSB1-LOV [4] and PpSB2-LOV proteins, respectively.

\section{References}

[1] A. Losi et al., Photochem. Photobiol. Sci. 7 (2008) 1168-78. doi:10.1039/b802472c.

[2] S. Endres et al., BMC Microbiol. 15 (2015) 30. doi:10.1186/s 12866-015-0365-0.

[3] F. Circolone et al., J. Mol. Biol. 417 (2012) 362-74. doi:10.1016/j.jmb.2012.01.056.

[4] K. Röllen et al., J. Mol. Biol. (2016). doi:10.1016/j.jmb.2016.05.027.

Keywords: LOV, photoreceptor, signal transduction, rotary switch 